\title{
Construcción cognitiva del espacio vectorial abstracto con estudiantes de Ingeniería
}

\section{Cognitive construction of abstract vector space with engineering students}

Deysi Margoth Guanga Chunata, ${ }^{1}$ Luis Enrique Sarmiento Torres, ${ }^{2}$ Marianela de Jesús Inca Chunata. ${ }^{3}$

\section{Recibido: 20-04-2019 / Revisado: 25-05-2019 /Aceptado: 28-06-2019/ Publicado: 30-07-2019}

\begin{abstract}
.
DOI: https://doi.org/10.33262/cienciadigital.v3i3.2.1.817

This paper describes the application and validation of a cognitive model for learning abstract vector spaces. The contribution that represents the association of real numbers, functions and any other element that could be consider as a vector, highlights the role played by the verification of the different algebraic structures, of the existence of a vector space, the linear transformation. The experimentation results obtained through with engineering students for the various applications developed in the classroom with a focus on developing solutions in the training area. The cognitive model shows a substantial improvement in the understanding of linear algebra subject.
\end{abstract}

Keywords: Cognitive model, Vector, Abstract vector space, Linear transform.

\section{Resumen.}

El presente trabajo describe la aplicación y validación de un modelo cognitivo para el aprendizaje de los espacios vectoriales abstractos. El aporte que representa el asociar los números reales, las funciones y cualquier otro elemento que pudiera ser considerado como un vector, destaca el papel que desempeña la comprobación de las diferentes estructuras algebraicas, de la existencia de un espacio vectorial, la transformación lineal. Los resultados obtenidos mediante la experimentación con estudiantes de ingeniería para las diversas aplicaciones desarrolladas en el aula de clase con enfoque al desarrollo de soluciones en el área de formación. El modelo cognitivo permite

\footnotetext{
${ }^{1}$ Escuela Superior Politécnica de Chimborazo, Chimborazo Ecuador, deysiguanga@espoch.edu.ec

${ }^{2}$ Petroamazonas EP, Sucumbíos, Ecuador. luis_sarmiento@petroamazonas.ec

${ }^{3}$ Secretaría Nacional de Educación Superior, Pichincha, Ecuador. minca@senescyt.gob.ec
} 
evidenciar una mejora sustancial en la comprensión de los contenidos de la asignatura algebra lineal

Palabras claves: Modelo cognitivo, Vector, Espacio vectorial abstracto, Transformación lineal.

\section{Introducción.}

Construir cognitivamente el espacio vectorial abstracto con estudiantes de ingeniería requiere un análisis retrospectivo en la historia de la epistemología para comprender la evolución de las construcciones matemáticas incorporadas a la estructura de espacio vectorial. Simultáneamente se requiere atender los procesos mentales involucrados en la estructura cognitiva del aprendizaje en estudiantes universitarios de ingeniería (Alfonso, A. 2012).

Es necesario asumir una posición frente a aquellos conceptos que definen la construcción de un espacio vectorial, sus elementos, axiomas, operaciones y transformaciones lineales. De este modo en la presente publicación se entenderá por $\mathrm{R} n$ al conjunto de todos los vectores cuyas componentes n-ésimas puedan ser concebidos como la colección de los n- números reales ordenados como primera, segunda, tercera... n-ésima componente. (Alfonso, A. 2012).

Se requiere también de un sistema coordenado que permita la representación geométrica de dichos elementos cartesianos (2 componentes), tridimensionales (tres componentes) y un sistema generador como un conjunto de vectores linealmente independientes que generan el espacio vectorial. (Kú, D. 2008). Estas nociones resaltan la estructura algebraica para los vectores del plano que se anclan a un origen como se ilustra en la Figura 1

Figura 1. Estructura algebraica

$$
\begin{aligned}
& \vec{u} \in R \vec{u}=(a) \\
& \vec{v} \in R^{2} \vec{v}=(a, b) \\
& \vec{w} \in R^{3} \vec{w}=(a, b, c) \\
& \vec{l} \in R^{4} \vec{l}=\left(x_{1}, x_{2}, x_{3}, x_{4}\right) \\
& \vdots \\
& \vec{\alpha} \in R^{n} \vec{\alpha}=\left(x_{1}, x_{2}, x_{3} \ldots, x_{n}\right)
\end{aligned}
$$

c.)vectores de componentes $1,2,3, \ldots n$ que pertenencen a los espacios vectoriales $R, R^{2}, R^{3}, R^{n}$ 
Figura 1. Sobre la concepción de plano cartesiano y plano vectorial

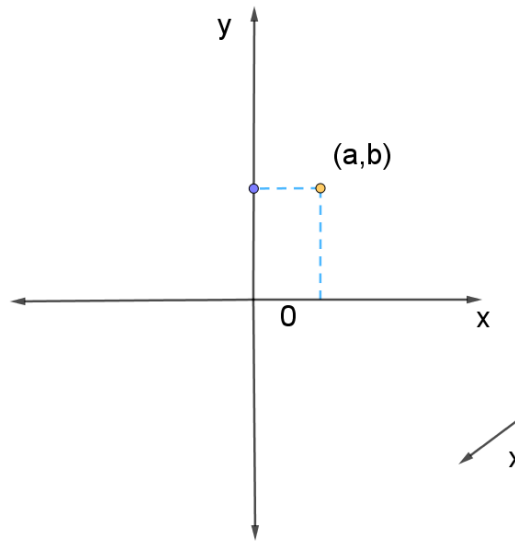

a.)Plano cartesiano $R^{2}$ par ordenado $(x, y)$
b.)PlanoTridimensional $R^{3}$

triada ordenada $(x, y, z)$

Fuente: Elaboración propia

Figura 2 Representación geométrica de los vectores de dos componentes
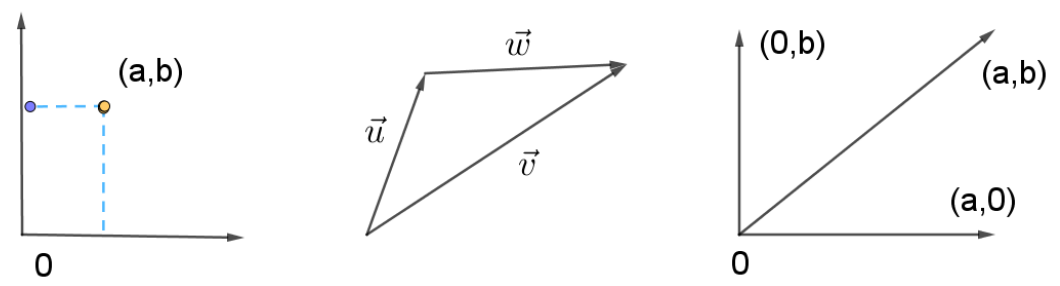

Fuente: Elaboración propia

La elección de estos diferentes escenarios se justifica, en parte, por la necesidad de explorar la influencia de las diferentes concepciones que existe sobre un conjunto ordenado de elementos, a la par con la idea segmento dirigido. (Arnon, I. 2014). Es imprescindible promover la transición colaborativa del aprendizaje entre los niveles medio y superior de la matemática para sustentar dicha construcción con base en un conocimiento previo más elemental. (Dorier, J. 2000).

\section{Metodología.}

Partiendo de las ideas de Descartes en 1637 se unificaron los criterios de la geometría, resaltan sobre las estructuras algebraicas -el concepto de grupo-que está relacionado con resolución de 
ecuaciones y la búsqueda de un cálculo geométrico que intentaron algunos matemáticos entre los siglos XVII y XIX para resolver problemas, estos hechos influyeron en la presentación de los axiomas Peano en 1888 de lo que hoy conocemos como espacio vectorial. Con respecto a la enseñanza de la geometría en los años sesenta, a la luz de la postura de algunos matemáticos franceses como Artín, Choquet y Revuz quienes destacan el rol del concepto de espacio vectorial en la caracterización del plano y el espacio geométrico, lo que pone en evidencia el tratamiento de la geometría a través de una estructura algebraica para alcanzar su visualización en el sentido de una construcción mental de objetos o procesos que un individuo asocia con objetos o medios externos tales como la pizarra o hasta un ordenador. (Dubinsky, E. 1996). Esta perspectiva permite apreciar el rol que desempeñaron los conceptos matemáticos con enfoque en la geometría, permitiendo de esta manera comprender los procesos de abstracción asociados con ella. Estos planteamientos constituyen la guía para la construcción cognitiva del espacio vectorial abstracto. Ruta que considera como punto de partida al producto cartesiano (Dorier, J. 2000).

A consecuencia de los efectos producidos por la reforma de las matemáticas modernas para poner en sintonía las estructuras matemáticas que promovió el grupo Bourbaki y las estructuras intelectuales que promocionó Piaget se impulsaron distintas iniciativas como las que se describen a continuación:

a. Un programa de investigación en álgebra lineal para indagar en el tipo de problemas asociados a la enseñanza - aprendizaje (Dorier, J. 2000).

b. Una reforma curricular para el álgebra lineal en Estados Unidos (Dubinsky, E. 1996).

c. El desarrollo de algunas concepciones teóricas para entender y explicar el porqué de las problemáticas en el aprendizaje de los conceptos ligados al álgebra lineal a nivel universitario (Dubinsky, E. 1996).

Las tres iniciativas antes mencionadas permiten situarnos en un panorama amplio respecto de la investigación y las innovaciones que desarrollaron distintos investigadores en torno a los conceptos del álgebra lineal.

La pregunta que orienta este trabajo es el siguiente:

¿Qué elementos matemáticos son necesarios y se evidencian como prerrequisitos para los estudiantes de ingeniería en la construcción cognitiva del espacio vectorial?

La Teoría APOE (Acrónimo de Acción, Proceso, Objeto y Esquema) es una teoría constructivista que toma como marco de referencia las ideas de Piaget respecto al desarrollo del conocimiento, fundamentalmente rescatando el concepto de abstracción reflexiva y el concepto de esquema. Dubinsky, quien desarrolla esta teoría, extiende el análisis cognitivo de conceptos matemáticos que se estudian de un nivel escolar, a un nivel de educación superior (Dubinsky, E. 1996). En la teoría APOE, las estructuras mentales que describen el proceso de construcción de un fragmento del conocimiento matemático constituido en cuatro 
categorías: acción, proceso, objeto y esquema. Conforme un individuo reflexiona y trabaja para comprender uno o varios conceptos matemáticos pone en juego mecanismos mentales: interiorización, coordinación, encapsulación y reversión, que son considerados casos particulares de la abstracción reflexiva [9]

Figura 3 Estructuras y mecanismos mentales para construir conceptos

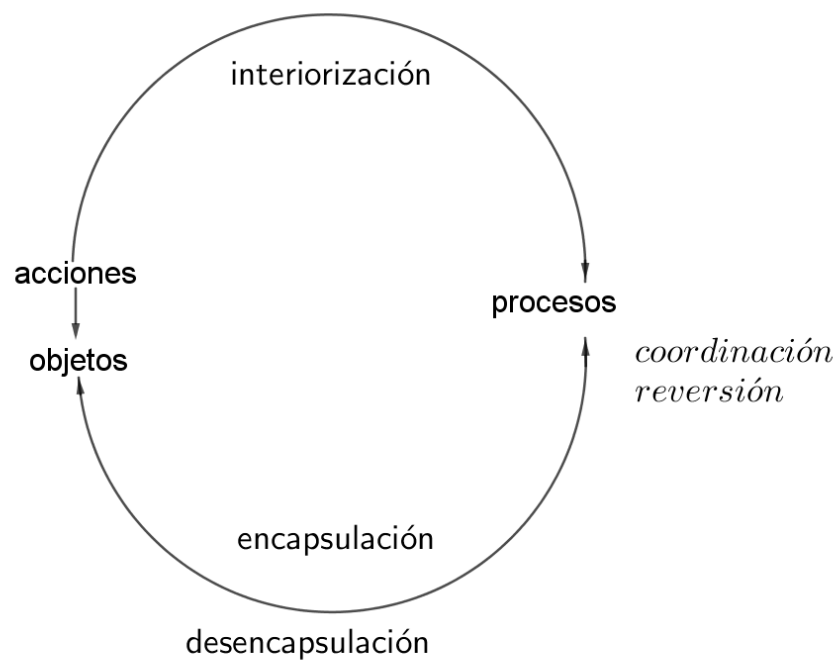

Fuente: Elaboración propia

Al considerar un objeto matemático se realizan acciones sobre objetos previamente construidos, relacionándolos con otros conceptos matemáticos que se toman como base para la construcción del nuevo. Las acciones se caracterizan por ser transformaciones que se realizan paso a paso, obedeciendo a estímulos que son o se perciben como externos (Dubinsky, E. 2001).

Un estudiante ha interiorizado una acción en un proceso si puede realizar una operación interna que hace (o imagina) esencialmente la misma transformación enteramente en su mente, sin necesariamente realizar todos los pasos específicos. (García, I. 2017). La teoría APOE proporciona un ciclo de investigación compuesto por tres componentes: el análisis teórico, el diseño y aplicación de instrumentos y el análisis y verificación de datos, el cual ha sido utilizado con éxito por el Grupo Rumec y otros investigadores (García, I. 2017).

\section{Resultados.}

Sobre la primera componente del método surge la interrogante ¿Qué es un vector? ¿Es un vector de dos dimensiones, por ejemplo, una flecha en el plano que describa con coordenadas por comodidad o es más bien un par de números reales que se pueden representar fácilmente como una flecha en el plano?, Puede que las dos cosas sean manifestaciones de algo más 
profundo, por un lado, definir un vector simplemente como una lista de números es directo y preciso, de este modo, nociones como vectores de 4 dimensiones e incluso de 100 parecen reales y concretas con las que se puede trabajar cómodamente, mientras que la idea de 4 dimensiones, en sí suena lejana y poco imaginable geométricamente y difícil de describir. Por otro lado, una intuición común entre los docentes que imparten la asignatura de álgebra lineal, especialmente cuando se interioriza el concepto de "cambio de base" es que se encuentra en un espacio que existe independientemente de las coordenadas que las asocie y que de hecho estas coordenadas son algo arbitrarias ya que varían en función de cómo se eligen los vectores de la base.

Temas básicos en álgebra lineal, como el de determinante o los vectores propios parecen indiferentes a la elección del sistema de coordenadas. El determinante representa cuánto varían las áreas bajo la transformación y los vectores propios son los que permanecen en el sub espacio que generan bajo esa misma transformación como lo muestra la Figura 4

Figura 4 El determinante y los valores propios no varían las áreas bajo la transformación.
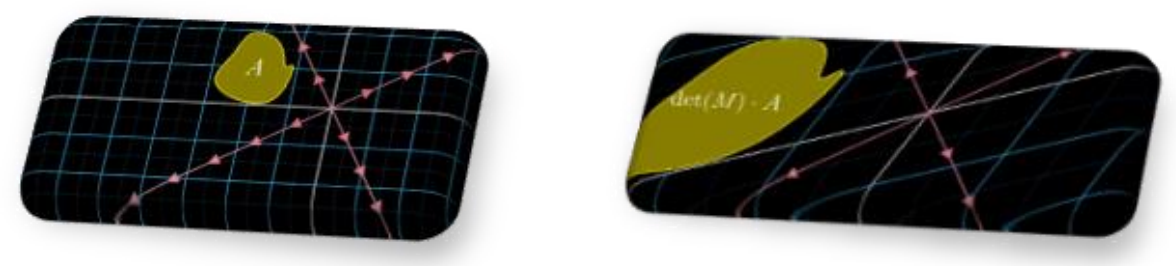

Fuente: Elaboración propia

Las dos propiedades son inherentes en el espacio y aun así es posible cambiar el sistema de coordenadas sin modificar ninguno de estos valores, pero si un vector no es una lista de números reales y su esencia se distingue como algo más espacial, esto conlleva a la reflexión sobre a qué se refiere la matemática cuando discurre sobre el término "espacio" o "espacial". Para ello se considera algo que no son ni "flechas", ni lita de números, pero que parece tener cualidades "vectoriales", las funciones que de hecho en el plano podrían parecer un tipo especial de vector.

Del mismo modo que se pueden sumar dos vectores, parece razonable que se puedan sumar dos funciones $f$ y $g$ para obtener una nueva función $(f+g)$ como lo muestra la Figura 5 . 
Figura 5 Adición de funciones

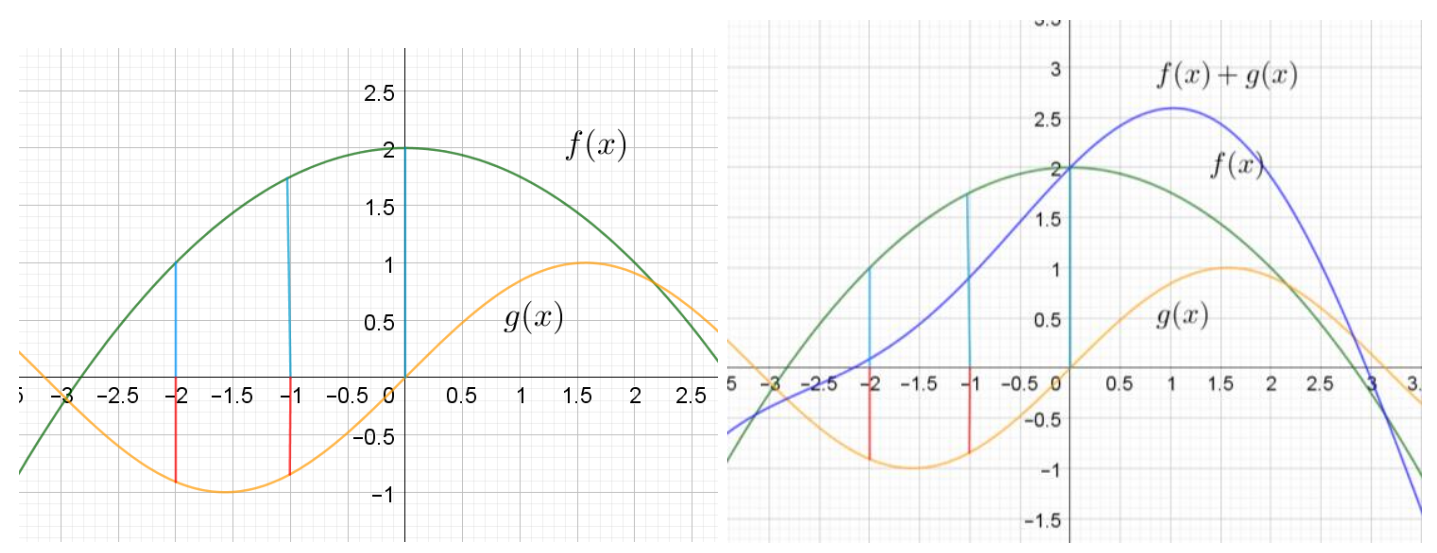

Fuente: Elaboración propia

La imagen de esta nueva función para cualquier valor de entrada, por ejemplo - 2 es la suma de las imágenes de $f$ cuando se evalúa -2 en cada una de ellas por separado. La imagen de esta nueva función para cualquier valor de entrada por ejemplo -2 es la suma de las imágenes de $f$ y $g$ cuando se evalúa en -2 en cada una de ellas por separado es decir:

$$
(f+g)(-2)=f(-2)+g(-2)
$$

En general la imagen de la función suma para cualquier valor de $x$ es la suma de las imágenes de $f(x)$ y $g(x)$. Este procedimiento es similar a la suma de vectores coordenada a coordenada, de algún modo existe un número infinito de coordenadas con las que trabajar.

$$
\left[\begin{array}{l}
x_{1} \\
y_{1} \\
z_{1}
\end{array}\right]+\left[\begin{array}{l}
x_{2} \\
y_{2} \\
z_{2}
\end{array}\right]=\left[\begin{array}{l}
x_{1}+x_{2} \\
y_{1}+y_{2} \\
z_{1}+z_{2}
\end{array}\right]
$$

De manera similar, resulta razonable la idea de multiplicar una función por un número real, bastará con multiplicar cada una de las imágenes por dicho número y otra vez es análogo a multiplicar los componentes de un vector coordenada a coordenada como lo muestra la Figura 6 
Figura 6 Producto de un escalar por una función

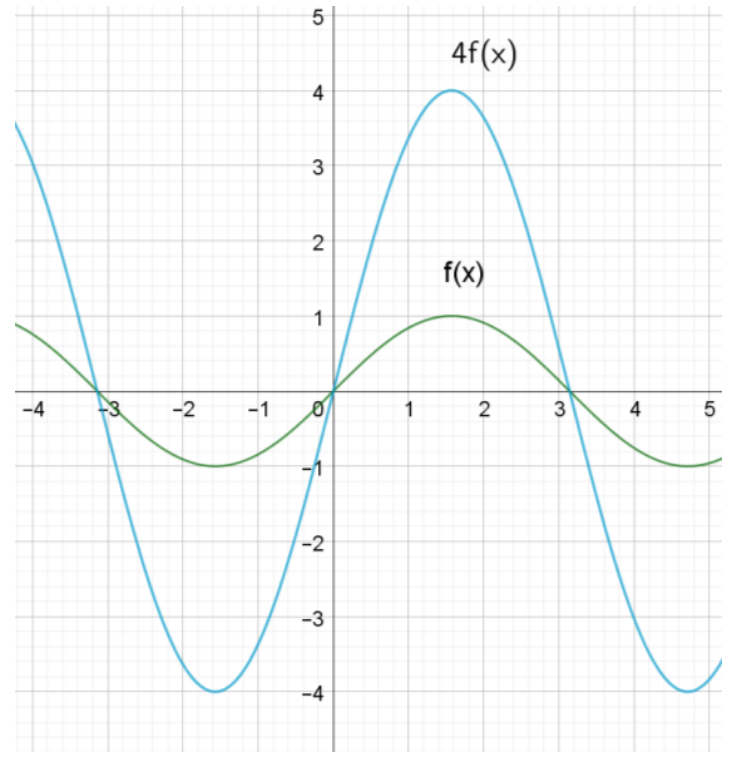

Fuente: Elaboración propia

Dado que los vectores admiten solamente ser sumados o multiplicados por un número real, existe una noción de transformación lineal para funciones, por ejemplo, una operación tal que dada una función devuelve otra proviene del cálculo y es la función derivada.

En ocasiones se utiliza el término operador en lugar de "transformación", sin embargo, el significado es el mismo. De manera natural surge la interrogante sobre el significado de la linealidad de la transformación lineal. La definición formal de linealidad es relativamente abstracta, una ventaja de la abstracción es que ésta permite aplicarla tanto en funciones como en vectores.

La transformación lineal es considerada como tal si satisface dos propiedades comúnmente descritas como aditividad y escalamiento.

$$
\begin{gathered}
T(\vec{u}+\vec{v})=T(\vec{u})+T(\vec{v}) \\
T(\alpha \vec{u})=\alpha T(\vec{u})
\end{gathered}
$$

Una de las consecuencias relevantes de estas dos propiedades que hace que la multiplicación matriz - vector sea posible, es que una transformación lineal queda totalmente determinada por la imagen de los vectores de la base. El hecho que todo vector pueda ser expresado como una suma de los vectores de la base multiplicados por un escalar real hace que la versión de 
transformada se reduzca a sumar y multiplicar por dichos números la versión transformada de los vectores de la base. Este concepto es válido para las funciones, por ejemplo, los estudiantes de cálculo usan continuamente la derivada de una función real, misma que es aditiva y tiene la propiedad del producto por un escalar, aunque quizá no familiaricen el concepto en estos términos en esa forma. Si se suman dos funciones y se hace su derivada, es lo mismo que hacer primero la derivada de cada una por separado y luego sumarlas.

$$
\frac{d}{d x}\left(x^{4}+3 x^{3}\right)=\frac{d}{d x}\left(x^{4}\right)+\frac{d}{d x}\left(3 x^{3}\right)
$$

De manera similar si se multiplica una función por un escalar y se hace su derivada, es lo mismo que hacer primero su derivada y luego multiplicar por el escalar.

$$
\frac{d}{d x}\left(3 x^{4}\right)=3 \frac{d}{d x}\left(x^{4}\right)
$$

Para evidenciar el paralelismo se describe la función derivada con una matriz. Esto resulta un poco enredado, ya que los espacios de funciones suelen tener dimensión infinita. Para ello se limita únicamente a la familia de los polinomios como $x^{2}+3 x+5$ o $4 x^{7}-5 x^{\wedge} 2$, cada uno de estos polinomios del espacio tendrá un número finito de componentes Pero el espacio en su totalidad incluye polinomios de grado arbitrariamente elevado. Lo primero es tomar coordenadas en este espacio que requiere escoger una base, al estar los polinomios ya escritos como una suma de potencias de la variable $x$ multiplicadas por un escalar parece natural elegir simplemente las potencias de x como las funciones de la base en otras palabras la primera función de nuestra base será la función constante $b_{0(x)}=1 \mathrm{La}$ segunda será $b_{1(x)}=0$ y después $b_{2(x)}=x^{\wedge} 2$ y después $b_{3(x)}=x^{\wedge} 3$ y así sucesivamente. El papel de estas funciones base será similar al papel de $i, j, k$ (vectores base del sistema tridimensional)

$$
\begin{aligned}
b_{0(x)} & =1 \\
1 x^{2}+3 x+5 \cdot x \quad b_{1(x)} & =x \\
b_{2(x)} & =x^{2}
\end{aligned}
$$


En el mundo de los vectores entendidos como flechas, como los polinomios pueden tener grado arbitrariamente elevado, el conjunto de función es base es infinito. Pero esto no es ningún problema, simplemente significa que cuando tratemos los polinomios como vectores tendrá un número infinito de coordenadas Un polinomio como $x^{2}+3 x+5$ por ejemplo se describiría como coordenadas 5,3,1 y después un número infinito de ceros esto se leería com que es 5 veces la primera función de la base, más tres veces la segunda función de la base intervienen en su construcción

$$
\left[\begin{array}{c}
5 \cdot 1 \\
+3 x \\
+1 x^{2} \\
+0 x^{3} \\
+0 x^{4} \\
\cdot \\
\cdot \\
\cdot
\end{array}\right]=\left[\begin{array}{c}
5 \\
3 \\
1 \\
0 \\
0 \\
\cdot \\
\cdot
\end{array}\right]
$$

El polinomio $4 x^{7}-5 x^{\wedge} 2$ tendría como coordenadas $(0,0,-5,0,0,0,0,4)$ y después un npumero infinito de ceros

$$
4 x^{7}-5 x^{2}=\left[\begin{array}{c}
0 \\
0 \\
-5 \\
0 \\
0 \\
0 \\
0 \\
4 \\
\cdot \\
\cdot \\
.
\end{array}\right]
$$

En general, al tener cada polinomio individualmente un número finito de términos, sus coordenadas serán un número finito de números seguido de un número infinito de ceros 


$$
a_{n} x^{n}+a_{n-1} x^{n-1}+\cdots a_{1} x+a_{0}=\left[\begin{array}{c}
a_{0} \\
a_{1} \\
a_{2} \\
\cdot \\
\cdot \\
\cdot \\
a_{n-1} \\
a_{n} \\
0 \\
\cdot \\
\cdot \\
\cdot \\
\cdot
\end{array}\right]
$$

En este sistema de coordenadas la derivada está descrita por una matriz infinita casi totalmente llena de ceros pero con enteros positivos en una de sus diagonales. Por ejemplo:

$$
\frac{d}{d x}=\left[\begin{array}{ccccc}
0 & 1 & 0 & 0 & \ldots \\
0 & 0 & 2 & 0 & \ldots \\
0 & 0 & 0 & 3 & \ldots \\
0 & 0 & 0 & 0 & \ldots \\
: & : & : & : & :
\end{array}\right]
$$

Tomando las coordenadas del polinomio $x^{3}+5 x^{2}+4 x+5$ para ponerlas a la derecha de la matriz, el único que contribuye a la primera coordenada es $1 \times 4$ que indica que el término constante del polinomio imagen será 4 . Esto corresponde al hecho que la derivada de $4 x$ es la constante 4, el único término que corresponde a la segunda coordenada del producto matriz- vector es $2 \times 5$ que significa que el coeficiente de delante de la $\mathrm{x}$ en la derivada es 10 esto corresponde a la derivada de $5 x^{2}$, de manera similar la tercera coordenada del producto matriz - vector se reduce a tomar $3 \times 1$, esto corresponde a la derivada de $x^{3}$ cuyo resultado es $3 x^{2}$ y después de este valor no habrá más que ceros.

$$
\begin{gathered}
\frac{d}{d x}\left(1 x^{3}+5 x^{2}+4 x+5\right)=3 x^{2}+10 x+4 \\
{\left[\begin{array}{ccccc}
0 & 1 & 0 & 0 & \ldots \\
0 & 0 & 2 & 0 & \ldots \\
0 & 0 & 0 & 3 & \ldots \\
0 & 0 & 0 & 0 & \ldots \\
: & : & : & : & :
\end{array}\right]\left[\begin{array}{c}
5 \\
4 \\
5 \\
1 \\
:
\end{array}\right]=\left[\begin{array}{c}
1 \cdot 4 \\
2 \cdot 5 \\
3 \cdot 1 \\
0 \\
:
\end{array}\right]}
\end{gathered}
$$


Lo que hace que estos razonamientos sean correctos es que la derivada es lineal. Para quien desee reflexionar sobre el tema, esta matriz se puede construir tomando la derivada de cada una de las funciones de la base y poniendo los resultados obtenidos a las columnas correspondientes.

$$
\frac{d}{d x} b_{2}(x)=\frac{d}{d x}\left(x^{2}\right)=2 x=\left[\begin{array}{l}
0 \\
2 \\
0 \\
0
\end{array}\right]
$$

Así pues de manera sorprendente, el producto matriz -vector y el cálculo de la función derivada, a priori cosas muy distintas, son simplemente miembros de la misma familia, En realidad muchos de los conceptos introducidos en este artículo con respecto a los vectores entendidos como flechas en el espacio como son el producto escalar, o los vectores propios, tiene análogos directos en el espacio de las funciones, aunque a veces sus nombres varían como por ejemplo producto interno, o funciones propias.

En general existe un sin número de elementos "vectoriales" que satisfacen las condiciones de escalabilidad y aditividad ya sea que éstos se identifiquen con las flechas en el espacio, listas de números, funciones o cualquier cosa que se desea definir, todas las herramientas del álgebra lineal involucrando vectores, transformaciones lineales y los demás axiomas, deberían ser aplicables.

Figura 6 Elementos vectoriales

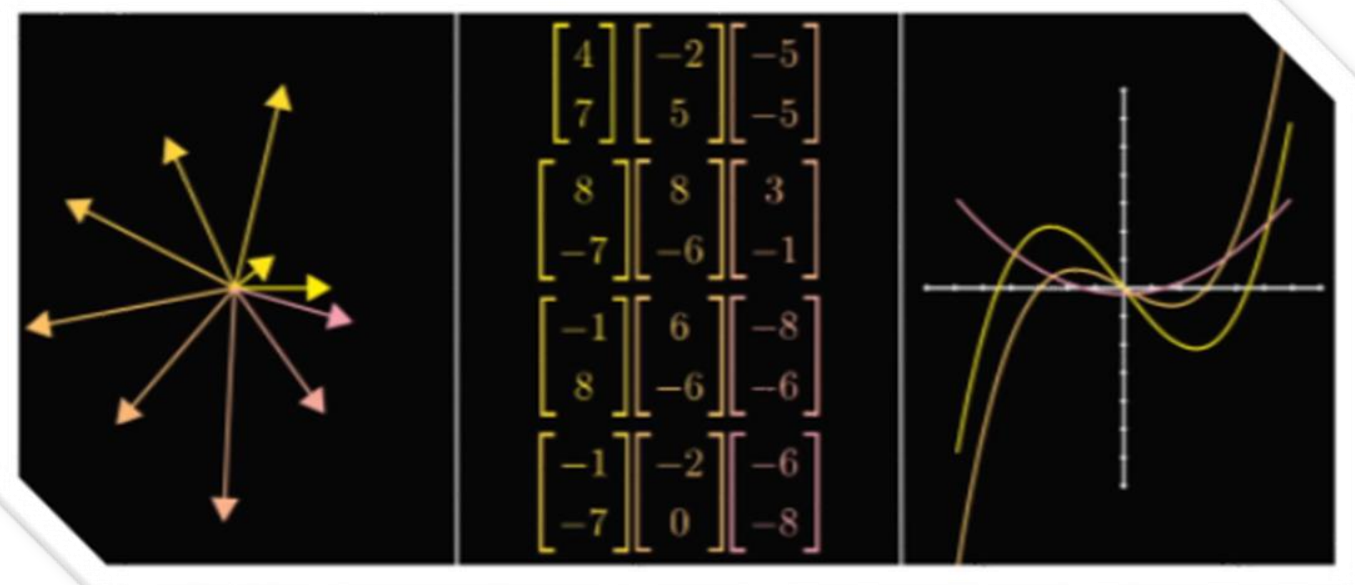

Fuente: Elaboración propia 
La matemática tiene la tarea de asentar las bases del álgebra lineal y de forma obligatoria se requiere que todas la definiciones y descubrimientos que se han realizado sirvan para aquello que sea un tanto vectorial con toda generalidad y no con casos concretos. Este conjunto de cosas vectoriales como las flechas en el espacio, listas de números o las funciones se llaman espacios vectoriales y se requiere que matemáticamente se pueda aseverar que cada uno de los posibles espacios vectoriales puedan ocurrir, de modo que el conjunto de reglas para la aditividad y al producto por un escalar se denominen axiomas y en la teoría moderna del álgebra lineal existen 8 de ellas que todo espacio vectorial debe satisfacer si se requiere que todas las herramientas y operaciones descritas funcionen.

Estos axiomas se enumeran a continuación:

1. $\vec{u}+(\vec{v}+\vec{w})=(\vec{u}+\vec{v})+\vec{w}$

2. $\vec{v}+\vec{w}=\vec{w}+\vec{v}$

3. $\exists \varepsilon \in$ del espacio vectorial tal que $\varepsilon+\vec{v}=\vec{v}$

4. Para cada $\vec{v}$ existe un $-\vec{v}$ tal que $\overrightarrow{v+(-\vec{v})}=\varepsilon$

5. $a(b \vec{v})=a b(\vec{v})$

6. $I \vec{v}=\vec{v}$

7. $a(\vec{v}+\vec{w})=a \vec{v}+a \vec{w}$

8. $(a+b) \vec{v}=a \vec{v}+b \vec{v}$

Esta lista describe los requisitos, para asegurar que la suma de vectores y su producto por escalares funcionen, sin pretender tampoco ser unas leyes fundamentales, sino más bien como un modo de comunicación para quienes desean aplicar estos resultados en nuevos tipos de espacios vectoriales. Si por ejemplo alguien define el extraño espacio vectorial del conjunto de "criaturas alfa" con algunas definiciones sobre su suma y su producto por escalares, estos axiomas servirían para comprobar qué deben cumplir para poder ser considerados como un espacio vectorial sin preocupaciones sobre lo extraño que podría resultar el conjunto, sino simplemente probar los resultados en términos de estos axiomas, de modo que, independientemente de las definiciones los resultados sean aplicables, incluso aunque se desconociera exactamente la situación. 
Como consecuencia los resultados serían abstractos, es decir únicamente en términos de estos axiomas más que en un tipo específico de vector, como flechas en el espacio o funciones. Por ejemplo, este es el motivo por el cual la mayoría de textos definen las transformaciones lineales en términos de aditividad y producto por un escalare, en lugar del tópico geométrico que versa sobre cuadrículas, sistemas coordenados paralelos y uniformes.

Aunque lo segundo sea más intuitivo y cognitivamente más útil para el estudiante. En la teoría moderna la forma que tomen los vectores no es relevante ya sea con listas de números, funciones, criaturas alfa, realmente no importa mientras cumplan las nociones de la suma y el producto por escalares.

\section{Análisis de los resultados}

Tabla 1. Resumen de la aplicación del método en dos grupos

- Caso 1

Fuente

- 15 Estudiantes de ingeniería en Telecomunicaciones.
- $\quad$ Caso 2

- 20 estudiantes de Ingeniería en Telecomunicaciones.

Técnica

- Aplicación del nuevo paradigma de espacios vectoriales abstractos

- Definición directa y textual de la construcción de espacios vectoriales

- $\quad$ Caso 1, Caso 2

Análisis - Del grupo de 35 estudiantes se aplicó un conjunto de problemas orientados de los a la identificación espacios vectoriales con elementos varios, 20 de datos estudiantes completaron la tarea establecida, 13 estudiantes corresponden al grupo en el que se aplicó el método.

Fuente: Elaboración propia

\section{Conclusiones}

- Para una mejor cognición se deben tratar los vectores como un elemento concreto y representable visualmente, como en el plano usando flechas desde el origen, considerando que a medida que el estudiante aprende más sobre álgebra lineal, es conveniente tener en cuenta que estos conocimientos son aplicables a un nivel mucho 
más general y que éste es el motivo por el cual los textos estudian el tema de un modo más abstracto

- El presente trabajo constituye una herramienta para el aprendizaje cognitivo de los espacios vectoriales abstractos. El resultado de la estrategia didáctica cognitiva permite a los alumnos su construcción al menos como proceso de aprendizaje dejando de lado la memorización.

\section{Referencias Bibliográficas.}

Alfonso, A. G., \& Becerra, J. R. (2012). Comprensión lectora y concepciones de estudiantes universitarios sobre enunciados matemáticos. Zona próxima: revista del Instituto de Estudios Superiores en Educación, (17), 2-23.

Arnon, I., Cottrill, J., Dubinsky, E., Oktaç, A., Roa Fuentes, S., Trigueros, M.\& Weller, K. (2014). APOS Theory: A framework for research and curriculum development in mathematics education. New York: Springer. doi: 10.1007/978-1-4614-7966-6 [ Links ]

Artigue, M. (2003). ¿Qué se puede aprender de la investigación educativa en el nivel universitario? Boletín de la Asociación Matemática Venezolana, 10(2), 117-134. [ Links ]

Dorier, J. L. (2000). Epistemological analysis of the genesis of the theory of vector spaces. In J-L. Dorier (Ed.), On the teaching of linear algebra (Vol. 23, pp. 3-81). Dordrecht: Springer. doi: 10.1007/0-306-47224-4_1 [ Links ]

Dorier, J. L. (Ed.). (1997). L’enseignement de l'algèbre linéaire en question. Grenoble: La Pensée Sauvage. [ Links ]

Dorier, J. L. \& Sierpinska, A. (2002). The teaching and learning of mathematics at university level. New ICMI Study Series, 7(3), 255-273. [ Links ]

Dubinsky, E. (1996). Aplicación de la perspectiva piagetiana a la educación matemática universitaria. Educación Matemática, 8(3), 24-41. [ Links ]

Dubinsky, E. \& MacDonald, M.A. (2001). APOS: A Constructivist Theory of Learning in Undergraduate Mathematics Education Research. In D. Holton et al. (Eds.), The Teaching and Learning of Mathematics at University Level: An ICMI Study, Kluwer Academic Publishers, pp. 275-282. [ Links ] 
García-Martinez, I. \& Parraguez, M. (2017). The basis step in the construction of the principle of mathematical induction based on APOS theory. The Journal of Mathematical Behavior, 46, 123-143. doi: 10.1016/j.jmathb.2017.04.001 [ Links ]

Kú, D., Trigueros, M., \& Oktaç, A. (2008). Comprensión del concepto de base de un espacio vectorial desde el punto de vista de la teoría APOE. Educación matemática, 20(2), 65-89. 


\section{PARA CITAR EL ARTÍCULO INDEXADO.}

Guanga Chunata, D., Sarmiento Torres, L., \& Inca Chunata, M. de J. (2019). Construcción cognitiva del espacio vectorial abstracto con estudiantes de Ingeniería. Ciencia Digital, 3(3.2.1), 258-274. https://doi.org/10.33262/cienciadigital.v3i3.2.1.817

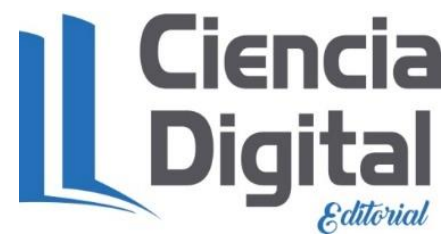

El artículo que se publica es de exclusiva responsabilidad de los autores y no necesariamente reflejan el pensamiento de la Revista Ciencia Digital.

El artículo queda en propiedad de la revista y, por tanto, su publicación parcial y/o total en otro medio tiene que ser autorizado por el director de la Revista Ciencia Digital.
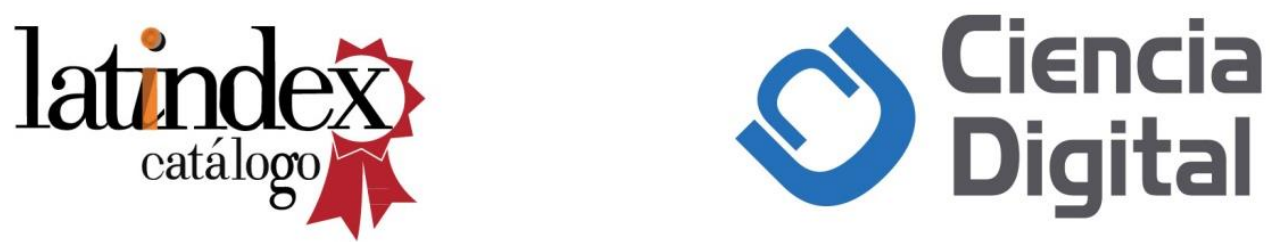